\title{
A microstructure study of post-growth thermally annealed InGaN/GaN quantum well structures of various well widths
}

\author{
Yen-Sheng Lin ${ }^{\mathrm{a}}$, Kung-Jen Ma ${ }^{\mathrm{b}}$, Yi-Yin Chung ${ }^{\mathrm{c}}$, Shih-Wei Feng ${ }^{\mathrm{c}}$, \\ Yung-Chen Cheng ${ }^{\mathrm{c}}$, En-Chiang Lin ${ }^{\mathrm{c}}$, C.C. Yang ${ }^{\mathrm{c}, *}$, \\ Cheng-Ta Kuo ${ }^{\mathrm{d}}$, Jian-Shihn Tsang ${ }^{\mathrm{d}}$ \\ ${ }^{a}$ Department of Electrical Engineering, Chinese Naval Academy, Kaohsiung, Taiwan, ROC \\ ${ }^{\mathrm{b}}$ Department of Mechanical Engineering, Chung Hua University, Hsinchu, Taiwan, ROC \\ ${ }^{\mathrm{c}}$ Department of Electrical Engineering, Graduate Institute of Electro-Optical Engineering, \\ National Taiwan University, 1, Roosevelt Road, Sec. 4, Taipei, Taiwan, ROC \\ d Advanced Epitaxy Technology Inc., Hsinchu Industrial Park, Taiwan, ROC
}

Received 21 October 2002; accepted 8 January 2003

Communicated by G.B. Stringfellow

\begin{abstract}
The microstructure variations upon post-growth thermal annealing at different temperatures of InGaN/GaN quantum well (QW) structures of different well widths are demonstrated. The high-resolution transmission electron microscopy images showed that with a small QW width $(2 \mathrm{~nm})$, thermal annealing tended to form quantum dots (QDs) of larger sizes $(3-5 \mathrm{~nm})$ to cover the designated QW layers. In this situation, strain might be well relaxed and quantumconfined Stark effect (QCSE) was reduced, resulting in a higher radiative efficiency. Also, with the formed QD structures, the expected stronger carrier localization (CL) effect might make a significant contribution to the enhanced radiative efficiency. On the other hand, with a large well width $(4 \mathrm{~nm})$, thermal annealing tended to make the QW boundaries clearer. In this situation, it seemed that either QCSE was enhanced or CL was reduced, resulting in degraded optical quality.
\end{abstract}

(C) 2003 Elsevier Science B.V. All rights reserved.

Keywords: A1. Segregation; A3. Quantum wells; B1. Nitrides

\section{Introduction}

Microstructure analyses have shown the formation of indium-aggregated quantum-dot (QD) clusters around the designated InGaN quantum

\footnotetext{
*Corresponding author. Tel.: + 886-2-2365-7624; fax: + 8862-2365-2637.

E-mail address: ccy@cc.ee.ntu.edu.tw (C.C. Yang).
}

well (QW) layers in an InGaN/GaN QW structure due to the large lattice constant mismatch $(\sim 11 \%)$ and hence low miscibility between $\mathrm{GaN}$ and InN [1-4]. Such cluster structures result in the effect of carrier localization (CL), which is believed helpful in enhancing radiative efficiency [5-7]. Also, the large lattice mismatch between an $\mathrm{InGaN}$ well and a $\mathrm{GaN}$ barrier leads to significant strains in the well layers. This strain distribution not only affects 
the aforementioned cluster formation, but also generates piezoelectric fields and hence the quantum confined Stark effect (QCSE) [8-11]. QCSE tilts the carrier potential and spatially separates electrons and holes, resulting in lower radiative efficiencies and longer photoluminescence (PL) decay times.

The sizes, shapes, compositions and distributions of the indium-aggregated clusters depend on the nominal indium content of $\mathrm{InGaN}$ and the degree of spinodal decomposition [4]. They also rely on the strain energy built during the growth of InGaN QW layers. Therefore, QW width becomes a crucial factor in cluster formation and the photon emission characteristics. It was reported that CL dominated in a narrow well structure and QCSE dominated in a wide well sample [12]. Besides the parameters above, the indium-aggregated structures are expected to be strongly related to the growth conditions. Hence, post-growth thermal annealing is quite effective in changing the cluster structures and photon emission properties [13-15]. Better indium-confined QD structures upon thermal annealing have been reported [15]. The same group has also observed the different thermal annealing effects on optical properties in InGaN/GaN QW structures of different QW widths.

In this paper, we report the different microstructure variations in these QW samples of different QW widths upon thermal annealing. In particular, we report the dependence of microstructure on annealing temperature. We found that under appropriate conditions, well-shaped QDs could be formed due to spinodal decomposition in a narrow QW sample, leading to radiative efficiency improvement. On the other hand, in a large QW width sample, thermal annealing led to clearer QW boundaries. However, it resulted in optical quality degradation.

This paper is organized as follows: In Section 2, sample structures and experimental procedures are discussed. The high-resolution transmission electron microscopy (HRTEM) images of various samples are described in Section 3 . Then, optical properties are briefly reported in Section 4. Finally, conclusions are drawn in Section 5.

\section{Sample preparation and experimental procedures}

The samples were grown on $c$-plane sapphire with metal organic chemical vapor deposition. Following the deposition of $30 \mathrm{~nm}$ GaN buffer layer and a $2.3 \mu \mathrm{m}$ GaN layer, QW structures consisting of five pairs of $\operatorname{In}_{0.15} \mathrm{Ga}_{0.85} \mathrm{~N}$ well and $10 \mathrm{~nm}$-thick GaN barrier were grown. Three samples of different well widths at 2, 3, and $4 \mathrm{~nm}$ were prepared and referred to as samples w20, w30 and $\mathrm{w} 40$, respectively. The growth temperatures were $1010^{\circ} \mathrm{C}$ and $720^{\circ} \mathrm{C}$ for $\mathrm{GaN}$ and $\mathrm{InGaN}$, respectively. As-grown samples were thermally annealed in a quartz tube furnace at $800^{\circ} \mathrm{C}$, $850^{\circ} \mathrm{C}$ and $900^{\circ} \mathrm{C}$ in nitrogen ambient for $30 \mathrm{~min}$.

Cross-sectional samples were prepared in the conventional manner by grinding, dimpling and $\mathrm{Ar}^{+}$-ion milling with $6 \mathrm{kV}, 1 \mathrm{~mA}$ and an incident angle of $4^{\circ}$. Based on previous experiences with compounds containing indium, the ion-milling step was carried out with the specimen holder at the liquid nitrogen temperature in order to minimize ion beam damage. HRTEM investigations were performed with both $200 \mathrm{keV}$ Philips CM 200 and $300 \mathrm{keV}$ JEM 3010 microscopes. All the high-resolution micrographs were taken at Scherzer defocus and the samples were viewed along the $(11 \overline{2} 0)$ zone axis. The $300 \mathrm{keV}$ JEM 3010 microscope is equipped with a $2 k \times 2 k$ slowscan CCD camera and a Gatan Imaging Filter (GIF). Energy filtering (EF) images were recorded by using the cross correlation algorithm available in the Digital Micrograph software of the GIF. To remove the background contribution underneath the ionization edges and to obtain elemental maps, the jump-ratio method was used to avoid noise and artifacts. PL measurements were conducted with a $\mathrm{He}-\mathrm{Cd}$ laser at $325 \mathrm{~nm}$ for excitation.

\section{High-resolution transmission electron microscopy results}

Figs. 1(a)-(c) show the HRTEM images of the as-grown, $800^{\circ} \mathrm{C}$ annealed and $900^{\circ} \mathrm{C}$ annealed w20 samples, respectively. The as-grown sample demonstrates quite diffusive distribution of indium although the rough positions of QWs can still be 

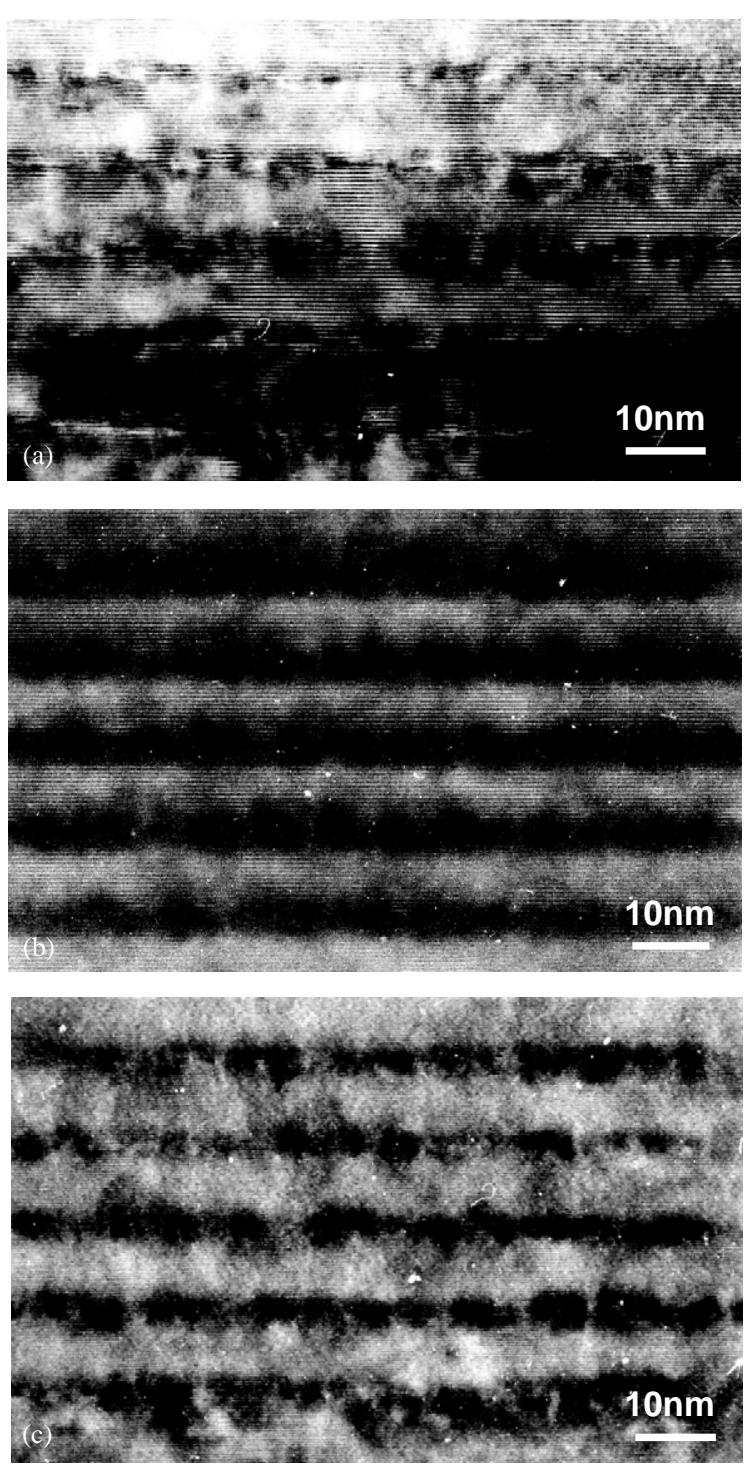

Fig. 1. HRTEM images of (a) the as-grown, (b) the $800^{\circ} \mathrm{C}$ annealed and (c) the $900^{\circ} \mathrm{C}$ annealed w 20 samples.

identified. After thermal annealing at $800^{\circ} \mathrm{C}$, indium distribution becomes much more confined around the QW layers. Such an indium gathering process ("up-hill" diffusion), caused by spinodal decomposition [4], resulted in quasi-regularly arranged QDs along the designated QW layers [15]. Their sizes, ranging from 3 to $5 \mathrm{~nm}$, are larger than the grown QW width ( $2 \mathrm{~nm}$ in this sample). Therefore, the distributed QDs cover the designated boundaries of QWs, if they exist. Such well- shaped QD structures seem to have well relaxed the built strains as the smooth contrast gradients imply. They indeed resulted in the highest photon emission efficiency in all the as-grown and annealed samples under study (see Fig. 4 and the discussions below). The HRTEM image of the $900^{\circ} \mathrm{C}$ annealed w20 sample (see Fig. 1(c)) showed more dispersed distribution of sharper QDs of various sizes and shapes although they were still distributed along the QW layers. In this case, the optical quality was degraded.

Figs. 2(a)-(c) show the HRTEM images of the as-grown, $800^{\circ} \mathrm{C}$ annealed and $900^{\circ} \mathrm{C}$ annealed w30 samples, respectively. In the as-grown w30, QW boundaries can be roughly identified although clusters of random nature tend to smear them out. Such a structure led to relatively lower photon emission efficiency, compared with the as-grown w2 2 (see Fig. 4). After thermal annealing at $800^{\circ} \mathrm{C}$, indium distribution tends to concentrate within the QW layers such that their boundaries become quite clear although several phase-separated clusters (fringe patterns) are observed. With such a structure, the optical quality was enhanced, compared with the as-grown sample. When the annealing temperature was increased to $900^{\circ} \mathrm{C}$, a significant change of microstructure was observed. As shown in Fig. 2(c), sharp QW boundaries can be seen with contrast distributions extending from the boundaries into barriers, particularly near the upper boundaries. Although the meanings of such contrast distributions require further exploration, significant strains near the clear QW interfaces are out of question. However, such a clear QW structure did not result in better optical quality. All the measured optical parameters (integrated PL intensity, PL spectral peak position and PL decay time) tended to return to the levels of the asgrown sample. The resultant strong QCSE can be the major cause for the lower photon emission efficiency.

Figs. 3(a)-(c) show the HRTEM images of the as-grown, $800^{\circ} \mathrm{C}$ annealed and $900^{\circ} \mathrm{C}$ annealed w40 samples, respectively. The as-grown sample demonstrates quite sharp QD structures of random nature. Although the QDs are distributed basically along the QW layers, their boundaries can hardly be seen. In spite of the possibly strong 

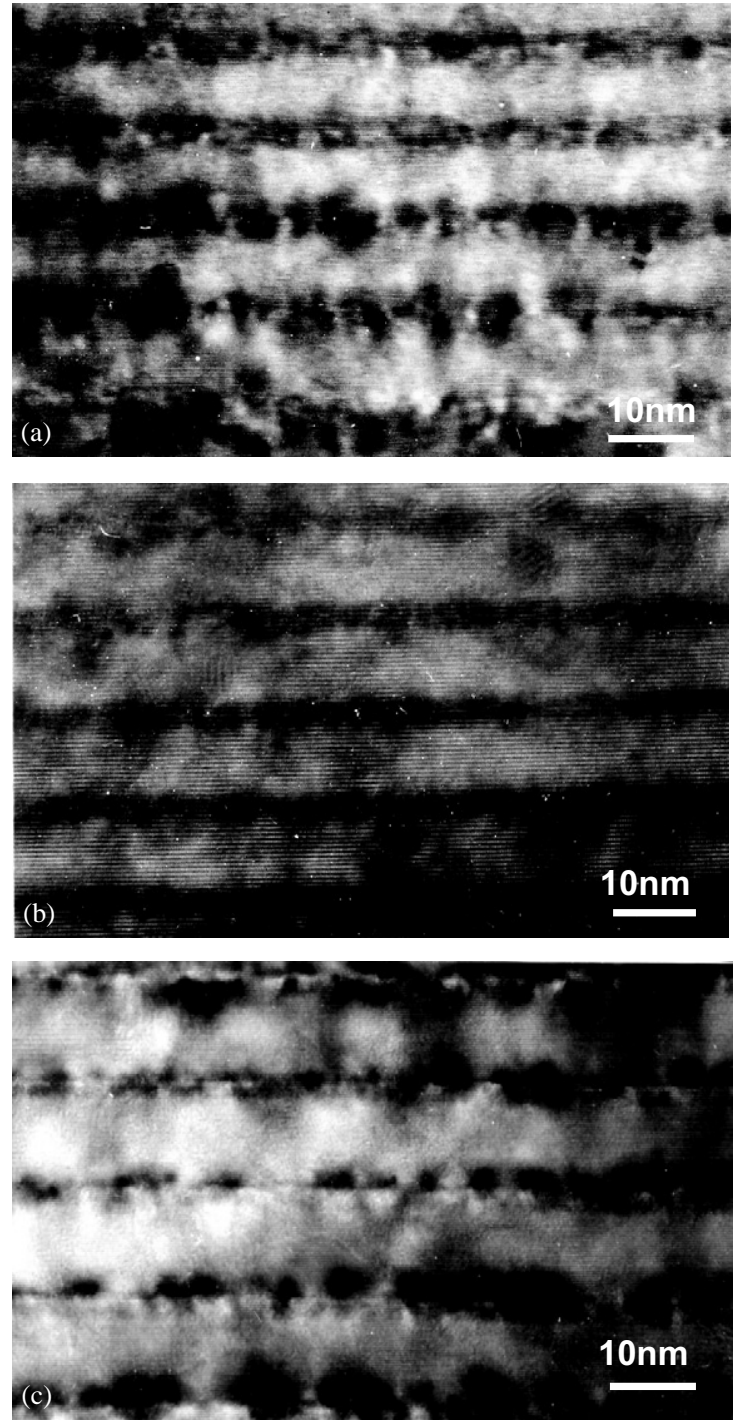

Fig. 2. HRTEM images of (a) the as-grown, (b) the $800^{\circ} \mathrm{C}$ annealed and (c) the $900^{\circ} \mathrm{C}$ annealed w30 samples.

$\mathrm{CL}$ in this sample, its photon emission efficiency is quite poor, compared with all other as-grown samples. After thermal annealing at $800^{\circ} \mathrm{C}$, QDs seemed to become smaller and sharper, as shown in Fig. 3(b). In this situation, line features of QW interfaces can be seen. As shown in Fig. 3(c), after annealing at $900^{\circ} \mathrm{C}$, the line features become quite clear. Such line features can be either the upper or lower boundaries of QWs. In this situation, strains seem to distribute on both sides of the line
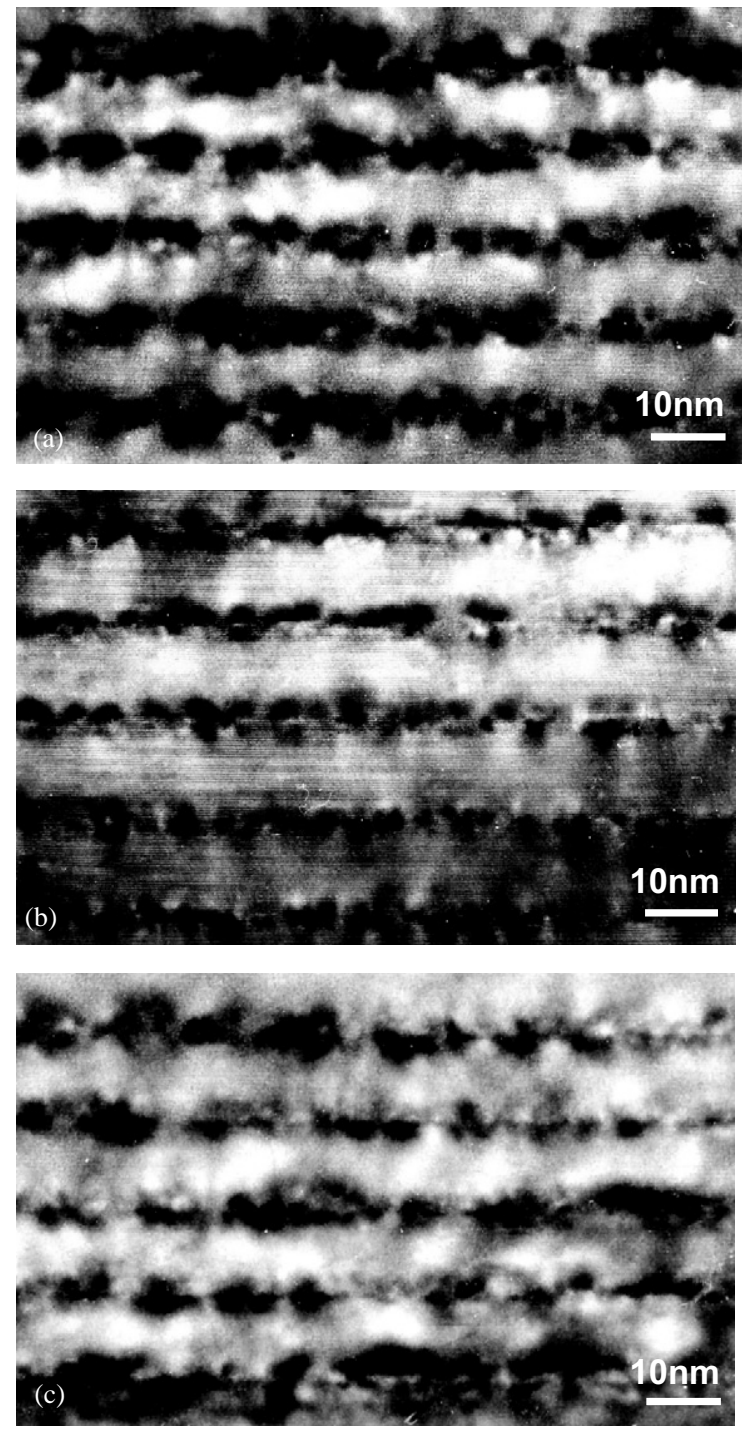

Fig. 3. HRTEM images of (a) the as-grown, (b) the $800^{\circ} \mathrm{C}$ annealed and (c) the $900^{\circ} \mathrm{C}$ annealed w40 samples.

features. The optical qualities of the annealed w40 samples were even worse than their as-grown counterpart. It is believed that QCSE plays the key role in determining the optical quality.

\section{Optical characterization results}

Optical characterization results were briefly mentioned above and are summarized as follows: 


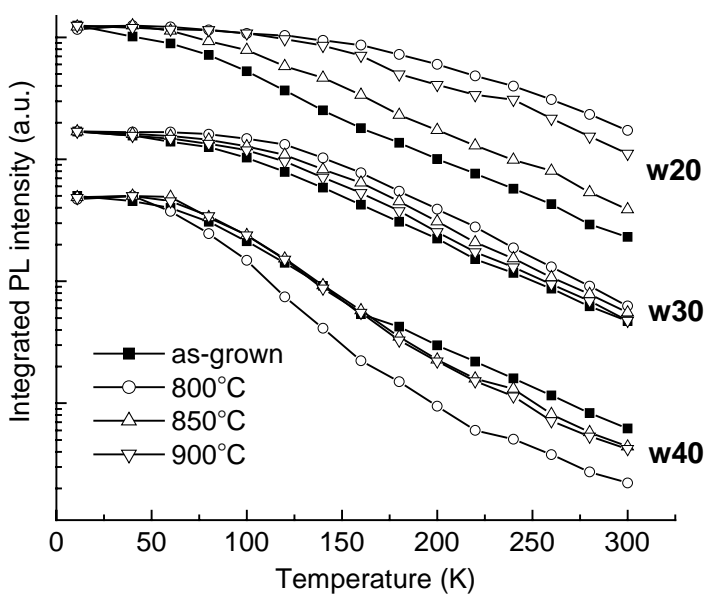

Fig. 4. Normalized integrated PL intensities versus temperature of the as-grown and annealed samples.

Upon thermal annealing, blue shift (red shift) in PL spectral peak position was observed in w20 (w30 and w40) with the largest shifts at $800^{\circ} \mathrm{C}$ annealing. When the annealing temperature increased, the blue or red shift diminished. Also, the photon emission efficiencies were enhanced in w20 and w30, and reduced in w40, as illustrated with the integrated PL intensity variations versus temperature in Fig. 4. Here, one can see that the radiative efficiency of the $800^{\circ} \mathrm{C}$ annealed w20 sample is 10 times higher than the as-grown sample. From Fig. 2(b) and other results from similar research of ours, we tend to conclude that the photon emission efficiency is optimized when the QD-like structure in Fig. 2(b) is formed. PL decay times of w20 (mainly in the high-temperature range) and w30 (mainly in the low-temperature range) were increased upon thermal annealing. The PL decay times of w40 became extremely long after thermal annealing and were out of our measurement capability. The variation trends of integrated PL intensity and PL decay time with annealing temperature were the same as that of PL peak position in all the three samples.

Because of the strong strain distributions around a QD, stacking faults and local distortions of atomic arrangement might exist. Fig. 5 shows an atomic-scale HRTEM of the $800^{\circ} \mathrm{C}$ annealed w40 sample. As indicated with circles, stacking faults or local distortions can be seen. Such defects

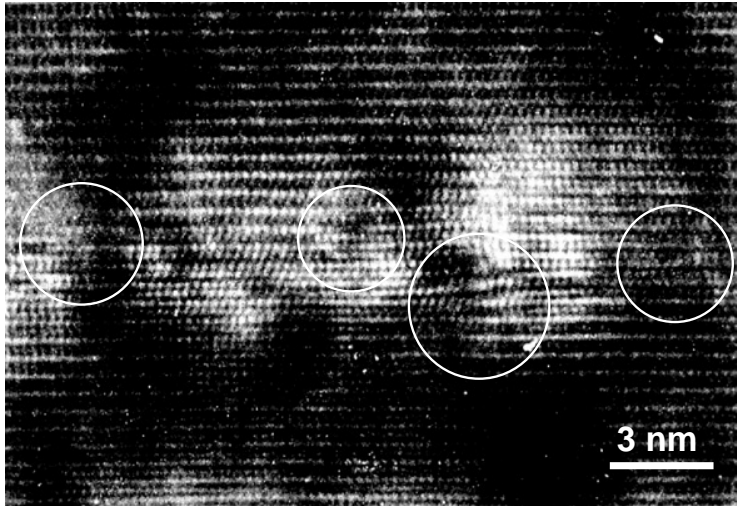

Fig. 5. Atomic-scale HRTEM image of the $800^{\circ} \mathrm{C}$ annealed w40 sample.

may represent a major cause of non-radiative recombination. Upon thermal annealing under a suitable condition, strains can be partly relaxed through spinodal decomposition and such defects can be annealed. Such a process is more likely to occur in a narrow well width sample, such as w20. However, in a larger well width sample, such as w40, strains around the sharp QDs seem to become stronger upon thermal annealing. Strain energy may be partly relaxed through the creation of more stacking faults and/or local distortions around QDs.

\section{Conclusions}

In summary, we have demonstrated the microstructure variations upon post-growth thermal annealing at different temperatures of $\mathrm{InGaN} /$ GaN QW structures of different QW widths. The HRTEM images showed that in a sample with a small QW width $(2 \mathrm{~nm})$, thermal annealing tended to form QDs of larger sizes $(3-5 \mathrm{~nm})$ such that arrayed QDs covered the designated QWs. In this situation, strain might be relaxed and quantumconfined Stark effect (QCSE) was reduced, leading to a higher radiative efficiency. Also, with the formed QD structures, the stronger CL effect might make a significant contribution to the enhanced radiative efficiency. On the contrary, in 
a sample with a large well width $(4 \mathrm{~nm})$, thermal annealing tended to make the QW boundaries clearer. This process was even more significant when thermal annealing took place at a higher temperature $\left(900^{\circ} \mathrm{C}\right)$. With the dominating QW structures, QCSE played the key role and the radiative efficiency was degraded. For a sample with the QW width in between $(3 \mathrm{~nm})$, the aforementioned QD and QW effects were supposed to mix.

\section{Acknowledgements}

This research was supported by National Science Council, The Republic of China, under the grants of NSC 90-2112-M-002-052, NSC 90-2215-E-002-027, and NSC 90-2215-E-002-041.

\section{References}

[1] Y. Narukawa, Y. Kawakami, M. Funato, S. Fujita, S. Fujita, S. Nakamura, Appl. Phys. Lett. 70 (1997) 981.

[2] M.D. McCluskey, L.T. Romano, B.S. Krusor, D.P. Bour, N.M. Johnson, S. Brennan, Appl. Phys. Lett. 72 (1998) 1730 .
[3] Y.S. Lin, K.J. Ma, C. Hsu, S.W. Feng, Y.C. Cheng, C.C. Liao, C.C. Yang, C.C. Chuo, C.M. Lee, J.I. Chyi, Appl. Phys. Lett. 77 (2000) 2988.

[4] I.H. Ho, G.B. Stringfellow, Appl. Phys. Lett. 69 (1996) 2701.

[5] Y. Narukawa, Y. Kawakami, S. Fujita, S. Fujita, S. Nakamura, Phys. Rev. B 55 (1997) R1938.

[6] T. Hino, S. Tomiya, T. Miyajima, K. Yanashima, S. Hashimoto, M. Ikeda, Appl. Phys. Lett. 76 (2000) 3421.

[7] Y.H. Cho, G.H. Gainer, A.J. Fischer, J.J. Song, S. Keller, U.K. Mishra, S.P. DenBarrs, Appl. Phys. Lett. 73 (1998) 1370.

[8] P. Riblet, H. Hirayama, A. Kinoshita, A. Hirata, T. Sugano, Y. Aoyagi, Appl. Phys. Lett. 75 (1999) 2241.

[9] S.F. Chichibu, A.C. Abare, M.S. Minsky, S. Keller, S.B. Fleischer, J.E. Bowers, E. Hu, U.K. Mishra, L.A. Coldren, S.P. DenBaars, T. Sota, Appl. Phys. Lett. 73 (1998) 2006.

[10] E. Berkowicz, D. Gershoni, G. Bahir, E. Lakin, D. Shilo, E. Zolotoyabko, A.C. Abare, S.P. Denbaars, L.A. Coldren, Phys. Rev. B 61 (2000) 10994.

[11] C.K. Choi, Y.M. Kwon, B.D. Little, G.H. Gainer, J.J. Song, Y.C. Chang, Phys. Rev. B 64 (2001) 245339.

[12] J. Bai, T. Wang, S. Sakai, J. Appl. Phys. 88 (2000) 4729.

[13] L.T. Romano, M.D. McCluskey, B.S. Krusor, D.P. Bour, C. Chua, S. Brennan, K.M. Yu, J. Crystal Growth 189/190 (1998) 33.

[14] C.C. Chuo, C.M. Lee, T.E. Nee, J.I. Chyi, Appl. Phys. Lett. 76 (2000) 3902.

[15] Y.S. Lin, K.J. Ma, C. Hsu, Y.Y. Chung, C.W. Liu, S.W. Feng, Y.C. Cheng, M.H. Mao, C.C. Yang, H.W. Chuang, C.T. Kuo, J.S. Tsang, T.E. Weirich, Appl. Phys. Lett. 80 (2002) 2571. 